\title{
Diurnal Variation of Nuclear Receptors in Mice with or without Fasting
}

\author{
Atsushi Kawase $^{1}$, Tetsuya Ohgami ${ }^{2}$, Iho Yoshida ${ }^{1}$, Yu Tsunokuni ${ }^{1}$, Masahiro Iwaki ${ }^{*}$ \\ ${ }^{1}$ Department of Pharmacy, School of Pharmacy, Kinki University, Osaka, Japan; ${ }^{2}$ Department of Physiology, Hyogo College of \\ Medicine, Nishinomiya, Japan. \\ Email: ${ }^{*}$ iwaki@phar.kindai.ac.jp
}

Received January $19^{\text {th }}, 2013$; revised March $1^{\text {st }}, 2013$; accepted April $7^{\text {th }}, 2013$

Copyright (C) 2013 Atsushi Kawase et al. This is an open access article distributed under the Creative Commons Attribution License, which permits unrestricted use, distribution, and reproduction in any medium, provided the original work is properly cited.

\begin{abstract}
Nuclear receptors such as pregnane $\mathrm{X}$ receptor (PXR) and constitutive androstane receptor (CAR) regulate the transcription of transporter and cytochrome P450 (CYP). The diurnal variation was observed in some transporters regulated by nuclear receptors. We investigated whether diurnal variation in PXR and CAR exists in mice. We also examined the effect of food intake on the diurnal rhythm of hepatic PXR and CAR using fed and fasted mice. In liver and small intestine of fed mice, the mRNA levels of PXR and CAR were unchanged between 7:00 AM and 7:00 PM. In contrast to fed mice, fasting mice partly exhibited the diurnal variation in PXR, not in CAR. The mRNA levels of PXR at 7:00 AM were significantly higher than that those at 7:00 PM in liver of fasting mice. These results indicated the different effects of fasting in mice on diurnal variation of PXR in each tissue.
\end{abstract}

Keywords: CAR; Circadian Rhythm; Diurnal Variation; Fasting; Nuclear Receptor; PXR

\section{Introduction}

The nuclear receptors pregnane X receptor (PXR, NR1I2: GenBank NM_010936) and constitutive androstane receptor (CAR, NR1I3: GenBank NM_009803) are involved in the primary response to xenobiotics and endogenous toxins. These receptors respond to ligands by activating the expressions of genes encoding enzymes involved in phase I (functionalization reactions) and phase II (conjugation reactions) metabolism and transporters [1-3]. Recent studies have shown that the gene expression of several transporters such as $\mathrm{H}^{+} /$peptide cotransporter PEPT1 [4] and P-glycoprotein [5] exhibited the diurnal variation. It is also reported that fasting changes both the quantity of cytochrome P450 (CYP) and the metabolic activity of rat hepatic microsomes, inducing CYP2B1, 2E1, and 3A2, and reducing CYP1A2 and $2 \mathrm{C} 11$ [6-9]. However, little is available on the correlation between the diurnal variation of transporter or CYP and that of nuclear receptor. The diurnal variation of PXR or CAR is an important factor to design the experiments for transporters or CYP if the diurnal variation were shown. It is known that the expression of transporters and CYP were affected on pathological condition. We

\footnotetext{
${ }^{*}$ Corresponding author.
}

also previously demonstrated that the mRNA or protein levels of transporters and CYP regulated by nuclear receptors were changed on arthritis condition [10,11].

In the present study, we investigated the diurnal variation of PXR and CAR in mice liver and small intestine. We also determined whether the fasting influences the diurnal variation of PXR and CAR. This information is important for the determination of alteration in transporters regulated by PXR and CAR.

\section{Materials and Methods}

\subsection{Animals}

Five to 6 weeks old male ddY mice were purchased from Japan SLC Co. (Shizuoka, Japan). Mice were housed in an air-conditioned room at $22^{\circ} \mathrm{C} \pm 0.5^{\circ} \mathrm{C}$ with a 12 -h lighting schedule (7:00 AM-7:00 PM) and were fed on the light/dark schedule for 1 week before they were divided into fed and fasted groups. Four different groups of mice were used in this study: 1) control group fed normal chow (MF, Oriental Yeast Co., Tokyo, Japan) sacrificed at 7:00 AM; 2) control group fed normal chow sacrificed at 7:00 PM; 3) fasted for 3 days sacrificed at 7:00 AM and 4) fasted for 3 days sacrificed at 7:00 PM. Water was available to all groups throughout the experiments. The 
experiments were approved by the Committee for the Care and Use of Laboratory Animals at Kinki University School of Pharmaceutical Science.

\subsection{Tissue Preparation}

The liver and small intestine were removed from mice with/without fasting after anesthesia by inhalation of ether and euthanasia by cervical dislocation. The small intestine was flushed with $50 \mathrm{~mL}$ of ice-cold saline, and was excised. The small intestine was a length of about $5 \mathrm{~cm}$ removed from about $5 \mathrm{~cm}$ below the ligament of Treitz. Each sample was preserved at $-80^{\circ} \mathrm{C}$ until use after flash freezing with liquid nitrogen.

\subsection{Real-Time Reverse Transcriptase Polymerase Chain Reaction}

Total RNA was extracted from approximately $100 \mathrm{mg}$ of each rat liver and small intestines using TRIzol Reagent (Invitrogen, Carlsbad, CA, USA) according to the manufacturer's instructions. Following RNase-free DNase I treatment (TaKaRa, Shiga, Japan), approximately $500 \mathrm{ng}$ total RNA, as evaluated by UV absorption at $260 \mathrm{~nm}$, was reverse-transcribed to complementary DNA (cDNA) using a PrimeScript-RT reagent Kit (TaKaRa) according to the manufacturer's instructions. The reactions were incubated for $15 \mathrm{~min}$ at $37^{\circ} \mathrm{C}$ and $5 \mathrm{~s}$ at $85^{\circ} \mathrm{C}$. The reverse-transcribed cDNA was used as template for realtime polymerase chain reaction (PCR). Amplification was performed in $50-\mu \mathrm{L}$ reaction mixtures containing $2 \times$ SYBR Premix Ex Taq (TaKaRa), $0.2 \mu \mathrm{M}$ primer set of target gene or ribosome $28 \mathrm{~S}$ ribosomal RNA (28S rRNA) as endogenous reference. Amplification and detection were performed with an ABI PRISM 7000 (Applied Biosystems, Foster City, CA, USA). The PCR reactions were incubated at $95^{\circ} \mathrm{C}$ for $10 \mathrm{~s}$, and amplified by a 40 three-step cycles at $95^{\circ} \mathrm{C}$ for $5 \mathrm{~s}, 55^{\circ} \mathrm{C}$ for $20 \mathrm{~s}$, and $72^{\circ} \mathrm{C}$ for $31 \mathrm{~s}$. The amount of $28 \mathrm{~S}$ rRNA in each sample was also measured for normalization. For all PCR amplifications, we used the following oligonucleotide sequences designed by Primer Express 2.0 (Applied Biosystems): PXR: 5'-CCCATCAACGTAGAGGAGGA-3' and 5'-GGGGGTTGGTAGTTCCAGAT-3'; CAR: 5'-GGAGGACCAGATCTCCCTTC-3' and 5'-ATTTCATTGCCACTCCCAAG-3'; and 28S rRNA: 5'-CGGCTCTTCCTATCATTGTG-3' and 5'-CCTGTCTCACGACGGTCTAA-3'. Data were analyzed using the ABI Prism 7000 SDS Software (Applied Biosystems) particularly for the multiplex comparative method. The relative quantitation of the amount of target mRNA in the tested tissue samples was accomplished by measuring Cycle thresholds $(\mathrm{Ct})$. To determine the quantity of the target gene-specific transcripts present in the liver and small intestines, their respective $\mathrm{Ct}$ values were first normalized by subtracting the $\mathrm{Ct}$ value obtained from the ribosome $28 \mathrm{~S}$ rRNA control $(\Delta \mathrm{Ct}=\mathrm{Ct}$, target $\mathrm{Ct}$, control). The concentration of gene-specific mRNA in the liver and small intestines of PM relative to each tissue of AM mouse was calculated by subtracting the normalized $\mathrm{Ct}$ values obtained for each tissue of AM mouse from those obtained from each tissue of PM mouse $(\Delta \Delta \mathrm{Ct}=\Delta \mathrm{Ct}, \mathrm{PM}-\Delta \mathrm{Ct}, \mathrm{AM})$ and the relative concentration was determined $\left(2^{-\Delta \Delta C t}\right)$.

\subsection{Statistical Analysis}

Significant differences between mean values of the gene expression levels were estimated using Student's unpaired t-test.

\section{Results and Discussion}

Figure 1 showed the diurnal variation of PXR and CAR in liver and small intestine derived from fed mice at 7:00 AM and 7:00 PM. PXR and CAR exhibited similar mRNA levels in liver and small intestine, suggesting that little diurnal variation of PXR (but not fasting) and CAR exists. To clarify the effect of fasting in mice on diurnal variation of nuclear receptors, the mRNA levels of PXR and CAR were determined in liver and small intestine of fasting mice. Figure 2 showed the diurnal variation of PXR and CAR in liver and small intestine derived from fasting mice at 7:00 AM and 7:00 PM. Interestingly, the mRNA levels of PXR exhibited diurnal variation by fasting for 3 days in liver. The PXR mRNA levels at 7:00 AM were significantly higher than those at 7:00 PM in liver. In contrast to PXR, the diurnal variation could not be observed in the CAR mRNA levels. Little change on diurnal variation of PXR and CAR was observed in small intestine. These results indicated that PXR and CAR exhibited little diurnal variation except for PXR in liver derived from fasting mice. The diurnal variation of transporters regulated by PXR and CAR could not be altered by the change in the mRNA levels of PXR or CAR under normal conditions. Therefore, diurnal variation in some transporters such as PEPT1 [4] or P-glycoprotein [5] could occur by change of other factors. These results also indicated that the consideration for diurnal variation in PXR and CAR is unimportant for experimental design under normal conditions. We assumed that fasting influenced the diurnal variation of nuclear recaptors because Pan et al. demonstrated that the expression of PEPT1 was affected by fasting [4]. In fasting condition, little diurnal variation of PXR or CAR was shown expect for PXR in liver. It is possible that the alterations in some factors such as lipid or glucose metabolisms under fasting condition affect the liver compared to the small intestine. However, it is unclear the precise mechanisms for diurnal variation induced in liver by fasting for 
Liver

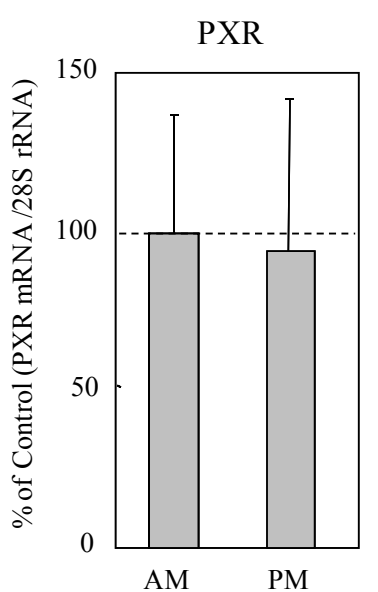

CAR

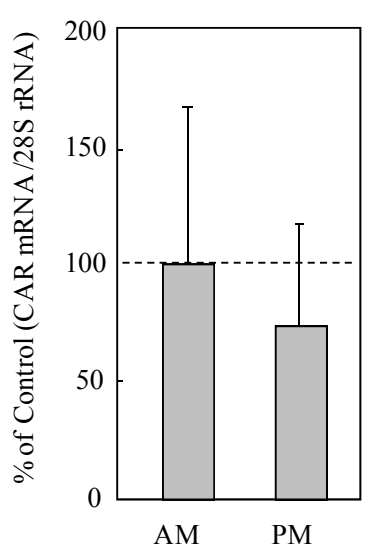

Small Intestine

Figure 1. Diurnal variation of PXR and CAR in liver and small intestine at 7:00 AM and 7:00 PM. The mRNA levels of PXR and CAR were determined in the livers using an RT-PCR method. The results are expressed as the mean \pm S.D. of each group following normalization by the corresponding level of $28 \mathrm{~S}$ rRNA $(\mathrm{n}=4)$.

Liver

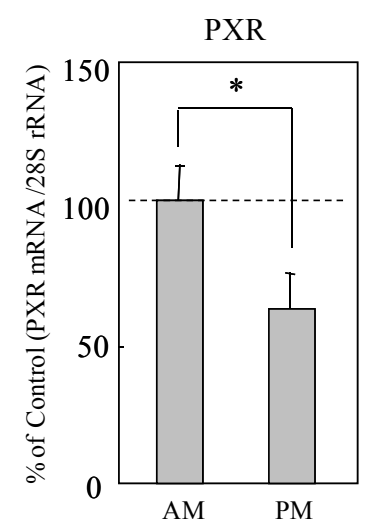

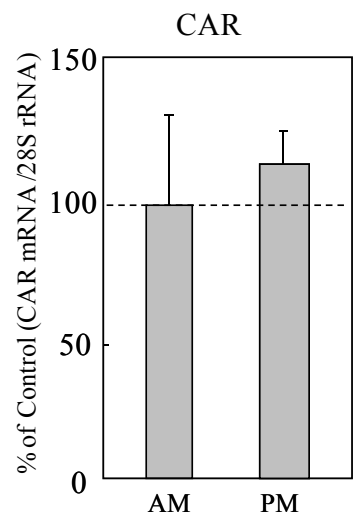
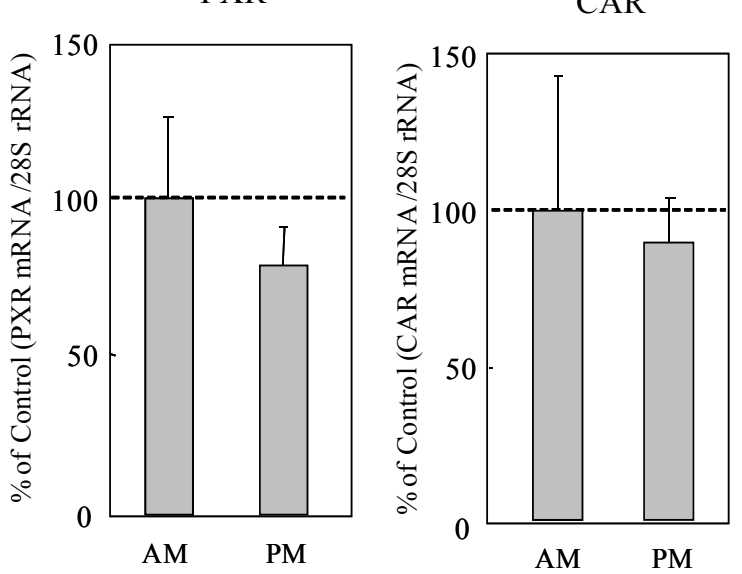

CAR , 
tors CAR, PXR, and PPAR," Archives of Biochemistry and Biophysics, Vol. 369, No. 1, 1999, pp. 11-23. doi:10.1006/abbi.1999.1351

[4] X. Pan, T. Terada, M. Okuda and K. Inui, "Altered Diurnal Rhythm of Intestinal Peptide Transporter by Fasting and Its Effects on the Pharmacokinetics of Ceftibuten," Journal of Pharmacology and Experimental Therapeutics, Vol. 307, No. 2, 2003, pp. 626-632. doi:10.1124/jpet.103.055939

[5] H. Ando, H. Yanagihara, K. Sugimoto, Y. Hayashi, S. Tsuruoka, T. Takamura, S. Kaneko and A. Fujimura, "Daily Rhythms of P-Glycoprotein Expression in Mice," Chronobiology International, Vol. 22, No. 4, 2005, pp. 655-665. doi:10.1080/07420520500180231

[6] C. S. Chaurasia, M. A. Alterman, P. Lu and R. P. Hanzlik, "Biochemical Characterization of Lauric Acid OmegaHydroxylation by a CYP4A1/NADPH-Cytochrome P450 Reductase Fusion Protein," Archives of Biochemistry and Biophysics, Vol. 317, No. 1, 1995, pp. 161-169. doi:10.1006/abbi.1995.1149

[7] J. Y. Hong, J. M. Pan, F. J. Gonzalez, H. V. Gelboin and C. S. Yang, "The Induction of a Specific Form of Cytochrome P-450 (P-450j) by Fasting," Biochemical and Biophysical Research Communications, Vol. 142, No. 3, 1987, pp. 1077-1083. doi:10.1016/0006-291X(87)91525-7

[8] S. Imaoka, Y. Terano and Y. Funae, "Changes in the
Amount of Cytochrome P450s in Rat Hepatic Microsomes with Starvation," Archives of Biochemistry and Biophysics, Vol. 278, No. 1, 1990, pp. 168-178. doi:10.1016/0003-9861(90)90245-T

[9] Q. Ma, G. A. Dannan, F. P. Guengerich and C. S. Yang, "Similarities and Differences in the Regulation of Hepatic Cytochrome P-450 Enzymes by Diabetes and Fasting in Male Rats," Biochemical Pharmacology, Vol. 38, No. 19, 1989, pp. 3179-3184. doi:10.1016/0006-2952(89)90611-4

[10] A. Kawase, Y. Tsunokuni and M. Iwaki, "Effects of Alterations in CAR on Bilirubin Detoxification in Mouse Collagen-Induced Arthritis," Drug Metabolism and Disposition, Vol. 35, No. 2, 2007, pp. 256-261. doi:10.1124/dmd.106.011536

[11] A. Kawase, I. Yoshida, Y. Tsunokuni and M. Iwaki, "Decreased PXR and CAR Inhibit Transporter and CYP mRNA Levels in the Liver and Intestine of Mice with Collagen-Induced Arthritis," Xenobiotica, Vol. 37, No. 4, 2007, pp. 366-374. doi:10.1080/00498250701230534

[12] J. M. Maglich, C. M. Stoltz, B. Goodwin, D. HawkinsBrown, J. T. Moore and S. A. Kliewer, "Nuclear Pregnane X Receptor and Constitutive Androstane Receptor Regulate Overlapping but Distinct Sets of Genes Involved in Xenobiotic Detoxification," Molecular Pharmacology, Vol. 62, No. 3, 2002, pp. 638-646. doi:10.1124/mol.62.3.638 